\section{PCR detection of a Maell polymorphism in the human major breakpoint cluster region (BCR)}

\section{J.S.McClure and C.E.Litz}

Department of Laboratory Medicine \& Pathology, Medical

School, Box 198, 420 Delaware St. SE, Minneapolis,

MN 55455, USA

Description/Primers: Nested primer pairs flanking the second intron of the breakpoint cluster region were constructed from the published cDNA sequence (1). The outer primer pair $5^{\prime} \mathrm{BCR}$ Exon 2 (5'-GTT TCA GAA GCT TCT CCC TG-3') and 3'BCR Exon 3 (5' - ACT CTG CTT AAA TCC AGT GG-3'), amplified a fragment of genomic DNA approximately $810 \mathrm{bp}$ in length. The inner primer pair, 3'BCR Exon 2 (5'-CGC TGA CCA TCA ATA AGG AA-3') and 5'BCR Exon 3 (5'-AGA AAC CCA TAG AGC CCC GG-3'), amplified a fragment approximately $730 \mathrm{bp}$ in length. Double stranded DNA amplified with the outer primer pair was subjected to asymmetric PCR using the inner primer pair. Sequencing reactions were performed using the Sequenase dideoxy sequencing kit with $\mathrm{S}^{35}$-dATP (US Biochemicals, Cleveland, $\mathrm{OH}$ ).

Sequence Data: Sequences in homozygotes revealed either an A or a G 85 bp 5' of the BCR BamHI site (Fig. 1-A, B). Heterozygotes demonstrated both bands at the corresponding position. The presence of a $\mathbf{G}$ in this position creates a sequence recognized by the restriction enzyme MaeII (ACGT).

Polymorphism: Digestion of the double-stranded DNA amplified with the outer primer pair (5'BCR Exon 2 and 3'BCR Exon 3) with MaeII revealed bands of $490 \mathrm{bp}$ and $320 \mathrm{bp}$ when the polymorphic site is present (Fig. 1-C). No other Maell site was present within the amplified region.

Frequency: The MaeII site has a gene frequency equal to 0.26 . Allele frequencies were calculated from 19 unrelated Caucasian individuals of predominantly northern European descent.

Chromosomal Localization: The BCR gene has been mapped to chromosome 22q11 (2).

References: 1) Heisterkamp,N. et al. (1985) Nature 315, 758-761. 2) Croce,C.M. et al. (1987) PNAS 84, 7174-7178.

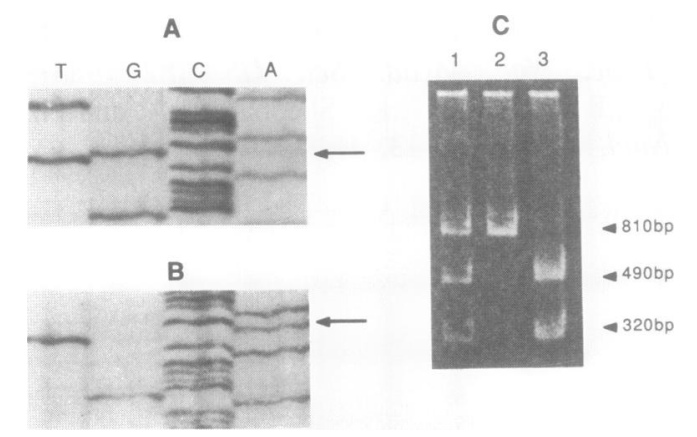

Figure 1-A and B: Partial PCR-derived BCR sequence from individuals homozygous for the presence (A) and absence (B) of the MaeII polymorphism. Arrows denote base change. C) Ethidium bromide stained polyacrylamide gel of amplified DNA digested with MaeII. Heterozygote (lane 1) and homozygote (lane 3) for MaeII polymorphism as well as homozygote (lane 2) for the absence of MaeII polymorphism are shown.

\section{A ScrFI polymorphism in the human ALPL gene}

T.Okuyama, N.Matsuo*, J.Kudoh ${ }^{1}$ and N.Shimizu'

Departments of Pediatrics and ${ }^{1}$ Molecular Biology, Keio

University School of Medicine, 35 Shinanomachi,

Shinjuku-ku, Tokyo 160, Japan

Source/Description: Amplification of the exon 12 untranslated region of the human liver/bone/kidney alkaline phosphatase (ALPL) gene $(1,2)$ was carried out by polymerase chain reaction (PCR) using the oligonucleotide 5'- AACTACGTCCCCCACGTGAT- $3^{\prime}$ as $5^{\prime}$ primer and the 5'-CTGGGCACGCTGGGTAGGAT-3' as $3^{\prime}$ primer. PCR was carried out by denaturation at $95^{\circ} \mathrm{C}$ for $1 \mathrm{~min}$, annealing at $62^{\circ} \mathrm{C}$ for $1 \mathrm{~min}$, and extending at $72^{\circ} \mathrm{C}$ for $2 \mathrm{~min}$. The amplified product was subsequently digested with ScrFI and analyzed on 5\% polyacrylamide gel in $90 \mathrm{mM}$ Tris-borate buffer (pH 8.3).

Polymorphism: ScrFI identifies two alleles $400 \mathrm{bp}(\mathrm{C1})$ and 300 bp (C2).

Frequency: 18 unrelated Japanese:

$\mathrm{A} 1=0.22$

$\mathrm{A} 2=0.78$

Not Polymorphic For: HhaI, HinfI, MspI.

Chromosomal Location: ALPL has been localized to 1p36.1- p34 (3).

Mendelian Inheritance: Co-dominant segregation was observed in 3 families.

References: 1) Weiss,M.J. et al. (1988) J. Biol. Chem. 263, 12002-12010. 2) Weiss,M.J. et al. (1986) Proc. Natl. Acad. Sci. USA 83, 7182-7186. 3) Smith,M. et al. (1988) Genomics 2, $139-143$. 